\title{
Some measure-valued Markov processes attached to occupation times of Brownian motion
}

\author{
CATHERINE DONATI-MARTIN and MARC YOR*
}

Laboratoire de Probabilités (UMR 7599), Université Paris VI, 4 place Jussieu, 75252 Paris

Cedex 05, France. E-mail: * secret@proba.jussieu.fr

We study the positive random measure $\Pi_{t}(\omega, \mathrm{d} y)=l_{t}^{B_{t}-y} \mathrm{~d} y$, where $\left(l_{t}^{a} ; a \in \mathbb{R}, t>0\right)$ denotes the family of local times of the one-dimensional Brownian motion $B$. We prove that the measure-valued process $\left(\Pi_{t} ; t \geqslant 0\right)$ is a Markov proces. We give two examples of functions $\left(f_{i}\right)_{i=1, \ldots, n}$ for which the process $\left(\Pi_{t}\left(f_{i}\right)_{i=1, \ldots, n} ; t \geqslant 0\right)$ is a Markov process.

Keywords: Brownian motion; local times; Markov processes

\section{Introduction}

Let $\left(B_{t}, t \geqslant 0\right)$ denote a one-dimensional Brownian motion, starting from 0 , and $\left(l_{t}^{y}\right.$; $y \in \mathbb{R}, t \geqslant 0)$ its family of local times. We denote by $\mathscr{F}_{t}$ the natural filtration of $B$.

Recently, a better understanding of an identity in law, originally due to Bougerol (1983), which involves an exponential functional of Brownian motion, was obtained by Alili et al. (1997) using the observation that if $\left(\xi_{t} ; t \geqslant 0\right)$ and $\left(\eta_{t} ; t \geqslant 0\right)$ are two independent Lévy processes, starting from 0 , then for any fixed $t \geqslant 0$,

$$
\int_{0}^{t} \mathrm{~d} \eta_{s} \exp \left(\xi_{s}\right) \stackrel{(\text { law })}{=} \exp \left(\xi_{t}\right) \int_{0}^{t} \mathrm{~d} \eta_{s} \exp \left(-\xi_{s}\right),
$$

and, moreover, the process

$$
Y_{t}^{(\xi, \eta) \stackrel{\text { def })}{=}} \exp \left(\xi_{t}\right) \int_{0}^{t} \mathrm{~d} \eta_{s} \exp \left(-\xi_{s}\right), \quad t \geqslant 0,
$$

is a Markov process. Equation (1.1) follows from the invariance by time reversal of the law of a Lévy process, and the Markov property of $Y^{(\xi, \eta)}$ is simply a consequence of the independence of the increments of $\xi$ and $\eta$. The importance of these generalized OrnsteinUhlenbeck processes was noticed and discussed in depth by de Haan and Karandikar (1989). Bougerol's identity,

$$
\text { for fixed } t, \quad \sinh \left(B_{t}\right) \stackrel{(\text { law })}{=} \int_{0}^{t} \mathrm{~d} C_{s} \exp \left(B_{S}\right)
$$


where $B$ and $C$ are independent Brownian motions, is then deduced easily taking in (1.2) for $\xi$ and $\eta$ two independent Brownian motions.

Here, we shall consider mainly the particular case where $\xi=B$ is a Brownian motion and $\eta_{t} \equiv t$, and pushing the preceding remark to the level of occupation times, we consider the positive random measure on $\mathbb{R}$

$$
\Pi_{t}(\omega, \mathrm{d} y)=l_{t}^{B_{t}-y} \mathrm{~d} y,
$$

which integrates positive functions $f: \mathbb{R} \rightarrow \mathbb{R}_{+}$as

$$
\Pi_{t}(f)=\int_{\mathbb{R}} f\left(B_{t}-z\right) l_{t}^{z} \mathrm{~d} z=\int_{0}^{t} f\left(B_{t}-B_{s}\right) \mathrm{d} s .
$$

In the case of an exponential function $f_{a}(x)=\exp (a x)$, the $\mathbb{R}_{+}$-valued process $\left(\Pi_{t}\left(f_{a}\right)\right.$, $t \geqslant 0$ ) is a Markov process (see (1.2)) which has been studied in Alili et al. (1997) and Carmona et al. (1997). On the other hand, for $f_{+}(x)=1_{x \geqslant 0}$, the process $\Pi_{t}\left(f_{+}\right)$was studied by Walsh (1993), where it is shown that $\left(\Pi_{t}\left(f_{+}\right), t \geqslant 0\right)$ is a Dirichlet process. Our aim here is to study the measure-valued process $\left(\Pi_{t} ; t \geqslant 0\right)$.

\section{A stochastic differential equation satisfied by $\left(\Pi_{t}, t \geqslant 0\right)$}

Proposition 2.1. The process $\left(\Pi_{t}, t \geqslant 0\right)$ is the unique solution (in the space $\mathscr{M}_{\mathrm{b}}(\mathbb{R})$ of bounded measures on $\mathbb{R}$ ) of the following $S D E$ : for every $f$ in $C_{\mathrm{b}}^{2}$,

$$
\Pi_{t}(f)=t f(0)+\int_{0}^{t} \mathrm{~d} B_{s} \Pi_{s}\left(f^{\prime}\right)+\frac{1}{2} \int_{0}^{t} \mathrm{~d} s \Pi_{s}\left(f^{\prime \prime}\right) .
$$

Proof. An application of Itô's formula to $f\left(B_{t}-B_{s}\right), t \geqslant s$, and Fubini's theorem show that $\left(\Pi_{t}, t \geqslant 0\right)$ solves the above stochastic differential equation (SDE). To prove uniqueness of the solutions of (2.1), we consider, for each $x \in \mathbb{R}$, the Fourier transform $\left(\Pi_{t}^{(x)}, x \in \mathbb{R}\right)$ of $\Pi_{t}$, that is, $\Pi_{t}^{(x)}=\int \Pi_{t}(\mathrm{~d} y) \exp (\mathrm{i} x y)$. Now, $\Pi_{t}^{(x)}$ solves a linear SDE; hence, $\left(\Pi_{t}\right)$ is the unique solution of the equation (2.1).

In the next corollary, we give some examples of functions $f$ (or $f_{1}, f_{2}, \ldots, f_{n}$ ) for which the process $\left(\Pi_{t}(f) ; t \geqslant 0\right)$ (or $\left.\left(\Pi_{t}\left(f_{i}\right)_{i=1, \ldots, n} ; t \geqslant 0\right)\right)$ is a Markov process.

Corollary 2.1. (a) For $f_{a}(x)=\exp (a x)$, the process $\left(\Pi_{t}\left(f_{a}\right)\right.$; $\left.t \geqslant 0\right)$ is an $\mathbb{R}_{+}$-valued Markov process (see (1.2)). More generally, for any $n \in \mathbb{N}$, and $a_{1}, \ldots, a_{n}$, the process $\left(\Pi_{t}\left(f_{a_{i}}\right)\right.$; $i \leqslant n)$ is an $n$-dimensional Markov process, whose infinitesimal generator coincides on $C^{2}\left(\mathbb{R}_{+}^{n}\right)$ with

$$
L=\frac{1}{2}\left(\sum_{i=1}^{n} a_{i}^{2} y_{i}^{2} \frac{\partial^{2}}{\partial y_{i}^{2}}+2 \sum_{i<j} a_{i} a_{j} y_{i} y_{j} \frac{\partial^{2}}{\partial y_{i} \partial y_{j}}\right)+\sum_{i=1}^{n}\left(\left(\frac{a_{i}^{2}}{2}+b_{i}\right) y_{i}+1\right) \frac{\partial}{\partial y_{i}} .
$$

(b) We set $\Pi_{t}^{(n)}=\Pi_{t}\left(P_{n}\right)$, where $P_{n}(x)=x^{n}$. Then, for every $n \in \mathbb{N},\left(\Pi_{t}^{(0)}, \ldots, \Pi_{t}^{(n)}\right)_{t \geqslant 0}$ 
constitutes an $\mathbb{R}^{n+1}$-valued Markov process, whose infinitesimal generator coincides on $C^{2}\left(\mathbb{R}^{n+1}\right)$ with

$$
L^{(n)}=\frac{1}{2}\left(\sum_{i=1}^{n} i^{2} x_{i-1}^{2} \frac{\partial^{2}}{\partial x_{i}^{2}}+2 \sum_{1 \leqslant i<j \leqslant n} i j x_{i-1} x_{j-1} \frac{\partial^{2}}{\partial x_{i} \partial x_{j}}\right)+\left(\frac{\partial}{\partial x_{0}}+\sum_{i=2}^{n} \frac{i(i-1)}{2} x_{i-2} \frac{\partial}{\partial x_{i}}\right) .
$$

Proof. This is just a consequence of formula (2.1).

Remarks. (a) We can write (2.1) formally as

$$
\left\{\begin{aligned}
\mathrm{d} \Pi_{t} & =\nabla^{*} \Pi_{t} \mathrm{~d} B_{t}+\left(\frac{1}{2} \Delta^{*} \Pi_{t}+\delta_{0}\right) \mathrm{d} t \\
\Pi_{0} & =0
\end{aligned}\right.
$$

where $\nabla$ is the operator $\partial / \partial x$ and $\Delta=\partial^{2} / \partial x^{2}$; that is, $\Pi_{t}$ solves a stochastic partial differential equation driven by a one-dimensional Brownian motion. This type of equation is well known and appears in filtering theory. We refer to Pardoux (1993) and Kallianpur (1996) for a review on stochastic partial differential equations and filtering theory.

(b) We can consider, more generally, the process $\left(\Pi_{t}^{A} ; t \geqslant 0\right)$ defined as

$$
\Pi_{t}^{A}(f)=\int_{0}^{t} \mathrm{~d} A_{s} f\left(B_{t}-B_{s}\right)
$$

where $\left(A_{t} ; t \geqslant 0\right)$ is a semimartingale, which is assumed to be independent of the Brownian motion $B$. Equation (2.1) becomes

$$
\Pi_{t}^{A}(f)=A_{t} f(0)+\int_{0}^{t} \mathrm{~d} B_{s} \Pi_{s}^{A}\left(f^{\prime}\right)+\frac{1}{2} \int_{0}^{t} \mathrm{~d} s \Pi_{s}^{A}\left(f^{\prime \prime}\right) .
$$

The simplest situation is $\mathrm{d} A_{t}^{(0)}=\delta_{0}(\mathrm{~d} t)$ which yields: $\Pi_{t}^{A^{(0)}}(f)=f\left(B_{t}\right)$. Note that all processes $\Pi_{t}^{A}$ satisfy the SDE:

$$
\sigma_{t}(f)=\sigma_{t}(1) f(0)+\int_{0}^{t} \sigma_{s}\left(f^{\prime}\right) \mathrm{d} B_{s}+\frac{1}{2} \int_{0}^{t} \sigma_{s}\left(f^{\prime \prime}\right) \mathrm{d} s .
$$

(c) Proposition 2.1 extends to the case where $B$ is a Brownian motion in $\mathbb{R}^{d}$ and $\left(\Pi_{t} ; t \geqslant 0\right)$ solves the follwing SDE: for $f \in C^{2}\left(\mathbb{R}^{d}\right)$,

$$
\Pi_{t}(f)=t f(0)+\int_{0}^{t} \Pi_{s}(\nabla f) \mathrm{d} B_{s}+\frac{1}{2} \int_{0}^{t} \mathrm{~d} s \Pi_{s}(\Delta f) .
$$

More generally, we can extend Proposition 2.1 to the $\mathbb{R} \times \mathscr{C}_{\mathrm{b}}(\mathbb{R})$-valued process $\left(B_{t}, \Pi_{t}\right)$.

Proposition 2.2. $\left(B_{t}, \Pi_{t} ; t \geqslant 0\right)$ is a continuous Markov process, with state space $\mathbb{R} \times \mathscr{C l}_{\mathrm{b}}(\mathbb{R})$ and is the solution of the following $S D E:$ for $f \in C^{2}(\mathbb{R}), g \in C^{2}(\mathbb{R})$, 


$$
\begin{aligned}
g\left(B_{t}\right) \Pi_{t}(f)= & \int_{0}^{t} \mathrm{~d} B_{s}\left(\Pi_{s}\left(f^{\prime}\right) g\left(B_{s}\right)+\Pi_{s}(f) g^{\prime}\left(B_{s}\right)\right) \\
& +\int_{0}^{t}\left(\frac{1}{2} \Pi_{s}(f) g^{\prime \prime}\left(B_{s}\right)+\frac{1}{2} \Pi_{s}\left(f^{\prime \prime}\right) g\left(B_{s}\right)+\Pi_{s}\left(f^{\prime}\right) g^{\prime}\left(B_{s}\right)+f(0) g\left(B_{s}\right)\right) \mathrm{d} s .
\end{aligned}
$$

This is immediate, using (2.1) and Itô's formula.

\section{Semigroup and generator of the process $\left(\Pi_{t} ; t \geqslant 0\right)$}

First, we introduce some notation. If $f: \mathbb{R} \rightarrow \mathbb{R}^{+}$is measurable, we denote by $\Phi_{f}$ the function on $\mathscr{C}_{\mathrm{b}}^{+}(\mathbb{R})$ defined by

$$
\Phi_{f}(v)=\exp (-\langle v, f\rangle)
$$

If $\Phi: \mathscr{C}_{\mathrm{b}}^{+}(\mathbb{R}) \rightarrow \mathbb{R}$, we set

$$
D \Phi(v)=\lim _{x \rightarrow 0} \frac{1}{x}\left(\Phi\left(\tau_{x} v\right)-\Phi(v)\right)
$$

when the limit exists and $\left\langle\tau_{x} v, f\right\rangle=\langle v, f(x+\cdot)\rangle$. Finally, $\Lambda_{t}$ denotes the occupation measure of $B$, that is,

$$
\Lambda_{t}(f)=\int_{0}^{t} f\left(B_{S}\right) \mathrm{d} s .
$$

Proposition 3.1. $\left(\Pi_{t} ; t \geqslant 0\right)$ is a homogeneous Markov process with state space $\mathscr{L}_{\mathrm{b}}^{+}(\mathbb{R})$ whose semigroup $\left(Q_{t} ; t \geqslant 0\right)$ is given by:

$$
Q_{t}(\mu ; \mathrm{d} v)=P\left(\tau_{B_{t}} \mu+\Lambda_{t} \in \mathrm{d} v\right) .
$$

The generator $\mathscr{B}$ of $\left(\Pi_{t} ; t \geqslant 0\right)$ coincides, on the functions $\Phi_{f}$, with

$$
\mathscr{b} \Phi_{f}(\mu)=\frac{1}{2} D^{2}\left(\Phi_{f}\right)(\mu)-f(0) \Phi_{f}(\mu)
$$

The resolvent of the semigroup $Q_{t}$ satisfies

$$
\begin{aligned}
U_{p}\left(\Phi_{f}\right)(\mu) & =\int_{0}^{\infty} \exp (-p t) Q_{t} \Phi_{f}(\mu) \mathrm{d} t \\
& =\int_{\mathbb{R}} \exp (-\langle\mu, f(x+\cdot)\rangle) U^{f}(p ; x) \mathrm{d} x,
\end{aligned}
$$

where the function $U(x):=U^{f}(p ; x)$ is the unique solution of the differential equation

$$
\frac{1}{2} U^{\prime \prime}(x)=(p+f(x)) U(x),
$$

subject to the condition that $U^{\prime}(x)$ exists for $x \neq 0$ and is bounded, that $U$ vanishes at $\pm \infty$ and that $U^{\prime}(0+)-U^{\prime}(0-)=-2$.

For fixed $t$, the law of $\Pi_{t}$, or equivalently the law of the process $\left\{l_{t}^{B_{t}-y} ; y \in \mathbb{R}\right\}$, has 
been described by Leuridan (1998); see also related work by Pitman (1998; 1999) who concentrates on the law of $\Pi_{t}$, conditionally on $B_{t}=b$, that is, the law of Brownian bridge local times. We note that although the equation satisfied by $\left(\Pi_{t}\right)$ is quite simple, the law of its marginal for fixed time $t$ is quite complicated, as shown in these papers.

Proof of Proposition 3.1. We note that the natural filtration $\mathscr{F}_{t}^{\Pi}$ of $\left(\Pi_{t}\right)$ is equal to the filtration of $B$, since for $f_{1}(x)=x$,

$$
\Pi_{t}\left(f_{1}\right)=\int_{0}^{t} s \mathrm{~d} B_{s}, \quad \text { and thus } \quad B_{t}=\int_{0}^{t} \frac{\mathrm{d} \Pi_{s}\left(f_{1}\right)}{s} .
$$

Furthermore,

$$
\begin{aligned}
& \mathrm{E}\left(\Phi_{f}\left(\Pi_{t+s}\right) \mid \mathscr{F}_{s}\right) \\
& =\mathrm{E}\left(\exp \left(-\int_{0}^{t+s} f\left(B_{t+s}-B_{u}\right) \mathrm{d} u\right) \mid \mathscr{F}_{s}\right) \\
& \quad=\mathrm{E}\left(\exp \left(-\int_{0}^{s} f\left(B_{t+s}-B_{s}+B_{s}-B_{u}\right) \mathrm{d} u\right) \exp \left(-\int_{s}^{t+s} f\left(B_{t+s}-B_{u}\right) \mathrm{d} u\right) \mid \mathscr{F}_{s}\right) .
\end{aligned}
$$

We introduce $\hat{B}_{v}=B_{v+s}-B_{s} .\left(\hat{B}_{v} ; v \geqslant 0\right)$ is a Brownian motion independent of $\mathscr{F}_{s}$. Thus,

$$
\mathrm{E}\left(\Phi_{f}\left(\Pi_{t+s}\right) \mid \mathscr{F}_{s}\right)=\hat{\mathrm{E}}\left(\exp \left(-\int_{0}^{s} f\left(\hat{B}_{t}+B_{s}(\omega)-B_{u}(\omega)\right) \mathrm{d} u\right) \exp \left(-\int_{0}^{t} f\left(\hat{B}_{t}-\hat{B}_{u}\right) \mathrm{d} u\right)\right),
$$

where the expectation is taken with respect to $\hat{B}$. Therefore,

$$
\begin{aligned}
Q_{t}\left(\Phi_{f}\right)(\mu) & =\mathrm{E}\left(\exp \left(-\left\langle\mu, f\left(B_{t}+\cdot\right)\right\rangle\right) \exp \left(-\int_{0}^{t} f\left(B_{s}\right) \mathrm{d} s\right)\right) \\
& =\mathrm{E}\left(\exp \left(-\left\langle\tau_{B_{t}} \mu+\Lambda_{t}, f\right\rangle\right)\right)=\mathrm{E}\left(\Phi_{f}\left(\tau_{B_{t}} \mu+\Lambda_{t}\right)\right) .
\end{aligned}
$$

This gives formula (3.1).

By definition of $\mathscr{B}$,

$$
\mathscr{B}\left(\Phi_{f}\right)(\mu)=\lim _{t \rightarrow 0} \frac{1}{t}\left(Q_{t}\left(\Phi_{f}\right)(\mu)-\Phi_{f}(\mu)\right)
$$

By (3.1), $\mathscr{B}\left(\Phi_{f}\right)(\mu)=I+J$, with

$$
\begin{aligned}
& I=\lim _{t \rightarrow 0} \frac{1}{t} \mathrm{E}\left(\left(\exp \left(-\left\langle\mu, f\left(B_{t}+\cdot\right)\right\rangle\right)-\exp (-\langle\mu, f\rangle)\right) \times \exp \left(-\int_{0}^{t} f\left(B_{u}\right) \mathrm{d} u\right)\right) \\
& J=\lim _{t \rightarrow 0} \frac{1}{t} \exp (-\langle\mu, f\rangle) \mathrm{E}\left(\exp \left(-\int_{0}^{t} f\left(B_{u}\right) \mathrm{d} u\right)-1\right) .
\end{aligned}
$$

It follows that: 


$$
I=\frac{1}{2} e_{f}^{\prime \prime}(0), \quad \text { where } e_{f}(x)=\exp (-\langle\mu, f(x+\cdot)\rangle)
$$

and

$$
J=\exp (-\langle\mu, f\rangle) f(0) .
$$

Now, by an easy computation, we verify that

$$
e_{f}^{\prime \prime}(0)=\exp (-\langle\mu, f\rangle)\left(\left(\left\langle\mu, f^{\prime}\right\rangle\right)^{2}-\left\langle\mu, f^{\prime \prime}\right\rangle\right)=D^{2}\left(\Phi_{f}\right)(\mu),
$$

proving formula (3.2).

Equation (3.3) is a consequence of the Feynman-Kac formula (Kac 1949; Jeanblanc et al. 1997):

$$
\int_{0}^{\infty} \exp (-p t) \mathrm{E}\left(q\left(B_{t}\right) \exp \left(-\int_{0}^{t} f\left(B_{u}\right) \mathrm{d} u\right)\right) \mathrm{d} t=\int_{\mathbb{R}} q(x) U^{f}(p ; x) \mathrm{d} x
$$

(where $U\left(=U^{f}\right)$ is defined as in the proposition) and of equation (3.1). The function $U$ can also be expressed as (see Jeanblanc et al. 1997, (3.14)):

$$
U^{f}(p ; x)=2 \frac{\Phi^{f_{+}}(p ; x) 1_{x>0}+\Phi^{f_{-}}(p ; x) 1_{x<0}}{-\Phi^{f_{+}}(p ; 0+)-\Phi^{f_{-}}(p ; 0+)},
$$

where $f_{+}$is the restriction of $f$ to $\mathbb{R}_{+}$and $f_{-}(x)=f(-x), x \geqslant 0$, and for a measurable function $g: \mathbb{R}_{+} \rightarrow \mathbb{R}_{+}, \Phi^{g}(p ; x)$ denotes the unique bounded solution of the SturmLiouville equations,

$$
\frac{1}{2} \Phi^{\prime \prime}=(p+g) \Phi, \quad \Phi(0)=1 .
$$

Let $\theta_{p}$ be an exponential variable of parameter $p$, independent of $\left(B_{t}, t \geqslant 0\right)$. Formula (3.4) reflects the path decomposition of $\left(B_{t} ; t \leqslant \theta_{p}\right)$ at time $g_{\theta_{p}}$, the last zero of $B$ before $\theta_{p}$ (see Jeanblanc et al. 1997).

As in the previous section, we can extend Proposition 3.1 to the process $\left(B_{t}, \Pi_{t}\right)$.

Proposition 3.2. $\left(B_{t}, \Pi_{t} ; t \geqslant 0\right)$ is a homogeneous Markov process with state space $\mathbb{R} \times$ $\mathscr{C}_{\mathrm{b}}(\mathbb{R})$ whose semigroup $R_{t}$ is given by

$$
R_{t}((x, \mu) ;(\mathrm{d} y, \mathrm{~d} v))=P\left(x+B_{t} \in \mathrm{d} y ; \tau_{B_{t}} \mu+\Lambda_{t} \in \mathrm{d} v\right) .
$$

The proof is similar to the previous one.

\section{An intertwining relationship between two measure-valued Markov processes}

Many examples of pairs $\left(X_{t}\right)$ and $\left(Y_{t}\right)$ of Markov processes with respect to filtrations $\left(\mathscr{O}_{t}\right)$ and $\left(\mathscr{Y}_{t}\right)$ such that $\mathscr{H}_{t} \subset \mathscr{X}_{t}$ lead to intertwining relationships between the semigroups of $X$ and $Y$; see for example Pitman and Rogers (1981), Yor (1989), Carmona et al. (1998) and, more recently Matsumoto and Yor (1998) in connection with exponential Brownian 
functionals - in particular $X_{t}=\int_{0}^{t} \exp \left(B_{t}-B_{s}\right) \mathrm{d} C_{s}$ and $Y_{t}=\int_{0}^{t} \exp \left(B_{t}-B_{s}\right) \mathrm{d} s$, where $B$ and $C$ are two independent Brownian motions satisfy an intertwining relationship.

We are interested in the extension of this result to the Markov processes $\left(\Pi_{t}\right)_{t}$ and $\left(\Pi_{t}^{C}\right)_{t}$, where $\Pi_{t}^{C}(f)$ is defined by

$$
\Pi_{t}^{C}(f)=\int_{0}^{t} \mathrm{~d} C_{s} f\left(B_{t}-B_{s}\right), t \geqslant 0 .
$$

For fixed $t$, the variable $\Pi_{t}^{C}$ is a random linear functional on $\mathscr{S}$, the Schwartz space of rapidly decreasing functions, that is, for $\varphi, \psi \in \mathscr{S}$ and $a, b \in \mathbb{R}$,

$$
\Pi_{t}^{C}(a \varphi+b \psi)=a \Pi_{t}^{C}(\varphi)+b \Pi_{t}^{C}(\psi) \text { a.s. }
$$

Since $\Pi_{t}^{C}$ is continuous in probability on $\mathscr{S}$ (using $\left\|\Pi_{t}^{C}(f)\right\|_{2} \leqslant C_{t}\|f\|_{L^{2}(\mathbb{R})}$ ), $\Pi_{t}^{C}$ has a version with values in $\mathscr{S}^{\prime}$ (see Walsh 1986, Corollary 4.2). So, we can consider the process $\left(\Pi_{t}^{C} ; t \geqslant 0\right)$ as a $\mathscr{S}^{\prime}$-valued process. Obviously, the process $\left(\Pi_{t} ; t \geqslant 0\right)$ can also be considered as a $\mathscr{S}^{\prime}$-valued process.

As in the previous section, we can express the semigroup $Q_{t}^{C}$ of the process $\Pi_{t}^{C}$ by

$$
\begin{aligned}
Q_{t}^{C}\left(\Phi_{f}\right)(\mu) & =\hat{\mathrm{E}}\left(\exp \left(-\left\langle\mu, f\left(\hat{B}_{t}+\cdot\right)\right\rangle\right) \exp \left(-\int_{0}^{t} f\left(\hat{B}_{t}-\hat{B}_{u}\right) \mathrm{d} \hat{C}_{u}\right)\right) \\
& =\mathrm{E}\left(\exp \left(-\left\langle\mu, f\left(B_{t}+\cdot\right)\right\rangle\right) \exp \left(-\int_{0}^{t} f^{2}\left(B_{u}\right) \mathrm{d} u\right)\right)
\end{aligned}
$$

for $f \in \mathscr{S}$ and $\mu \in \mathscr{S}^{\prime}$.

Proposition 4.1. The semigroups $Q_{t}$ and $Q_{t}^{C}$ enjoy the intertwining relationship

$$
Q_{t} \mathscr{C l}=\mathscr{C l} Q_{t}^{C},
$$

where $\mathscr{C}$ is a Markov kernel from $\mathscr{S}^{\prime}$ to $\mathscr{S}^{\prime}$ defined on the functions $\Phi_{f}(f \in \mathscr{S})$ by

$$
\mathscr{L}\left(\Phi_{f}\right)(\mu)=\mathrm{E}\left(\exp \left(-\mu\left(f^{2}\right)^{1 / 2}\right) N\right)=\exp \left(\frac{1}{2} \mu\left(f^{2}\right)\right)
$$

in which $N$ denotes a standard Gaussian variable. In other words, $\mathscr{C l}(\mu, \mathrm{d} v)$ is a centred Gaussian measure over $\mathscr{S}^{\prime}$ with intensity $\mu$.

Sketch of proof. We define $\mathscr{G}_{t}=\sigma\left\{B_{u}, C_{u} ; u \leqslant t\right\}$. We compute the expression

$$
A=\mathrm{E}\left(\Phi_{f}\left(\Pi_{t+s}^{C}\right) \mid \mathscr{F}_{t}\right)
$$

first by conditioning with respect to $\mathscr{F}_{t+s}$. Now, conditionally to $\mathscr{F}_{t+s}$,

$$
\Pi_{t+s}^{C}(f) \stackrel{(\text { law })}{=}\left(\Pi_{t+s}\left(f^{2}\right)\right)^{1 / 2} N,
$$

where $N$ is a standard Gaussian variable, independent of $B$. Then, we obtain

$$
A=Q_{s}\left(\mathscr{L} \Phi_{f}\right)\left(\Pi_{t}\right) \text {. }
$$

On the other hand, by conditioning first with respect to $\mathscr{G}_{t}$, we find 


$$
A=\mathscr{L}\left(Q_{f}^{C}\left(\Phi_{s}\right)\right)\left(\Pi_{t}\right)
$$

\section{The process $\int_{0}^{t} f\left(B_{t}-B_{s}\right) \mathrm{d} B_{s}$}

It seems natural to extend the definition of the process $\Pi_{t}^{A}$ defined by (2.3) to the case where $A=B$. Since, for $t$ fixed, the process $\left(B_{t}-B_{s} ; s<t\right)$ is not $\mathscr{F}_{s}$-adapted, we must make precise the meaning of the stochastic integral $\int_{0}^{t} f\left(B_{t}-B_{s}\right) \mathrm{d} B_{s} .\left(B_{t}-B_{s} ; s \leqslant t\right)$ is $\mathscr{T}^{s}:=$ $\sigma\left\{B_{u}-B_{t} ; s \leqslant u \leqslant t\right\}$ adapted; therefore, we can define this integral as a backward Itô integral and we denote it by

$$
\int_{0}^{t} f\left(B_{t}-B_{s}\right) \mathrm{d}_{-} B_{s} .
$$

We recall briefly the definition of the backward integral: for an $\mathscr{T}^{s}$-measurable process $H_{s}$,

$$
\int_{0}^{t} \mathrm{~d}_{-} B_{s} H_{s} \stackrel{\text { def }}{=}-\int_{0}^{t} \mathrm{~d} \hat{B}_{s}^{(t)} H_{t-s}
$$

where $\hat{B}_{s}^{(t)}=B_{t}-B_{t-s}$, and on the right-hand side, the integral is a forward integral with respect to the Brownian motion $\hat{B}^{(t)}$.

Note that this integral coincides with the Skorohod integral (see Nualart and Pardoux 1988).

Proposition 5.1. The $\mathscr{S}^{\prime}$-valued process $\left(\Pi_{t}^{B} ; t \geqslant 0\right)$ defined by

$$
\Pi_{t}^{B}(f)=\int_{0}^{t} f\left(B_{t}-B_{s}\right) d_{-} B_{s}
$$

satisfies, for every $f$ in $C_{\mathrm{b}}^{2}$,

$$
\Pi_{t}^{B}(f)=B_{t} f(0)+\int_{0}^{t} \mathrm{~d} B_{s} \Pi_{s}^{B}\left(f^{\prime}\right)+\frac{1}{2} \int_{0}^{t} \mathrm{~d} s \Pi_{s}^{B}\left(f^{\prime \prime}\right) .
$$

Proof. We apply Itô's formula to $f\left(B_{t}-B_{s}\right)$ and we use the following Fubini-type identity (see Rosen and Yor 1991, (2.2) and (2.3)):

$$
\int_{0}^{t} \mathrm{~d}_{-} B_{s} \int_{s}^{t} \mathrm{~d} B_{u} \varphi\left(B_{u}-B_{s}\right)=\int_{0}^{t} \mathrm{~d} B_{u} \int_{0}^{u} \mathrm{~d}_{-} B_{s} \varphi\left(B_{u}-B_{s}\right) .
$$

Remark. We can also prove (5.1) without using (5.2). Take $f$ of the form

$$
f(x)=\int g(\xi) \exp (\mathrm{i} x \xi) \mathrm{d} \xi
$$

Then, 


$$
\begin{aligned}
X_{t} & :=\int_{0}^{t} \exp \left(\mathrm{i} \xi\left(B_{t}-B_{u}\right)\right) \mathrm{d}_{-} B_{u} \\
& =\exp \left(\mathrm{i} \xi B_{t}\right) \int_{0}^{t} \exp \left(-\mathrm{i} \xi B_{u}\right) \mathrm{d} B_{u}-i \xi \exp \left(\mathrm{i} \xi B_{t}\right) \int_{0}^{t} \exp \left(-\mathrm{i} \xi B_{u}\right) \mathrm{d} u
\end{aligned}
$$

using the well-known property for Skorohod integrals (see Nualart and Pardoux 1988):

$$
\delta(F u .)=F \delta(u .)-\int_{0}^{t} D_{t} F u_{t} \mathrm{~d} t .
$$

We now apply Itô's formula to $X_{t}$. Integrating then with respect to $g(\xi) \mathrm{d} \xi$ (and using a classical Fubini theorem) yields the result.

\section{A measure-valued process related to Pitman's theorem}

It is shown in Matsumoto and Yor (1998) that for $\lambda \in \mathbb{R}$, the process

$$
\exp \left(-\lambda B_{t}\right) \int_{0}^{t} \mathrm{~d} s \exp \left(2 \lambda B_{s}\right)
$$

is a Markov process with respect to its own filtration, a result from which one recovers asymptotically Pitman's celebrated theorem (see Pitman 1975).

By analogy with our present work, this prompted us to define a measure-valued process $\left(\tilde{\Pi}_{t}\right)$ by

$$
\tilde{\Pi}_{t}(f)=\int_{0}^{t} \mathrm{~d} s f\left(2 B_{s}-B_{t}\right)
$$

which satisfies the equation

$$
\tilde{\Pi}_{t}(f)=\int_{0}^{t} \mathrm{~d} s f\left(B_{s}\right)+\int_{0}^{t} \mathrm{~d} B_{s} \tilde{\Pi}_{s}\left(f^{\prime}\right)+\frac{1}{2} \int_{0}^{t} \mathrm{~d} s \tilde{\Pi}_{s}\left(f^{\prime \prime}\right) .
$$

However, the analogy with $\left(\Pi_{t}\right)$ cannot be pushed much further, as discussed in Matsumoto and Yor (1998), to which we refer the reader: in particular, $\left(\tilde{\Pi}_{t}\right)_{t}$ is not a Markov process. On the other hand, note how similar the equation (6.1) is to equation (2.1), the only change being that 'the given data' $t \delta_{0}$ has been changed in (6.1) into the occupation measure $\int_{0}^{t} \mathrm{~d} s \delta_{B_{s}}$.

\section{References}

Alili, A., Dufresne, D. and Yor, M. (1997) Sur l'identité de Bougerol pour les fonctionnelles du mouvement brownien avec drift. In M. Yor (ed.), Exponential Functionals and Principal Values Related to Brownian Motion, Bibl. Rev. Mat. Iberoamericana, pp. 3-14. Madrid: Revista Mathemática Iberoamericana.

Bougerol, P. (1983) Exemples de théorèmes locaux sur les groupes résolubles. Ann. Inst. H. Poincaré Probab. Statist., 19, 369-391. 
Carmona, P., Petit, F. and Yor, M. (1997) On the distribution and asymptotic results for exponential functionals of Lévy processes. In M. Yor (ed.), Exponential Functionals and Principal Values Related to Brownian Motion, Bibl. Rev. Mat. Iberoamericana, pp. 73-121. Madrid: Revista Mathemática Iberoamericana.

Carmona, P., Petit, F. and Yor, M. (1998) Beta-gamma random variables and intertwining relations between certain Markov processes. Rev. Mat. Iberoamericana, 14, 311-367.

de Haan, L. and Karandikar, R.L. (1989) Embedding a stochastic difference equation into a continuous-time process. Stochastic Process. Appl., 32, 225-235.

Jeanblanc, M., Pitman, J. and Yor, M. (1997) The Feynman-Kac formula and decomposition of Brownian paths. Comput. Appl. Math., 16, 27-52. Boston: Birkhäuser.

Kac, M. (1949) On the distributions of certain Wiener functionals. Trans. Amer. Math. Soc., 65, 1-13.

Kallianpur, G. (1996) Some recent developments in non linear filtering theory. In N. Ikeda, S. Watanabe, M. Fukushima and H. Kunita (eds), Itô's Stochastic Calculus and Probability Theory, pp. 157-170. Tokyo: Springer-Verlag.

Leuridan, C. (1998) Le théorème de Ray-Knight en un temps fixe. In J. Azéma, M. Emery, M. Ledoux and M. Yor (eds), Séminaire de Probabilités XXXII, Lecture Notes in Math. 1686, pp. 376-396. Berlin: Springer-Verlag.

Matsumoto, H. and Yor, M. (1998) Some extensions of Pitman's theorem involving exponential functionals via generalized inverse gaussian distributions. Preprint.

Nualart, D. and Pardoux, E. (1988) Stochastic calculus with anticipating integrands. Probab. Theory Related Fields, 78, 535-581.

Pardoux, E (1993) Stochastic partial differential equations: a review. Bull. Sci. Math., 117, 29-47.

Pitman, J. (1975) One-dimensional Brownian motion and the three-dimensional Bessel process. Adv. Appl. Probab., 7, 511-526.

Pitman, J. (1999) The SDE solved by local times of a Brownian excursion or bridge derived from the height profile of a random tree or forest. Ann. Probab., 27, 261-283.

Pitman, J. (1999) The distribution of local times of a Brownian bridge. In J. Azéma, M. Emery, M. Ledoux and M. Yor (eds), Séminaire de Probabilités XXXIII, Lecture Notes in Math. 1709. Berlin: Springer-Verlag.

Pitman, J. and Rogers, L.C.G. (1981) Markov functions. Ann. Probab., 9, 573-582.

Rosen, J. and Yor, M. (1991) Tanaka formulae and renormalization for triple intersections of Brownian motion in the plane. Ann. Probab., 19, 142-159.

Walsh, J. (1986) An introduction to stochastic partial differential equations. In P.L. Hennequin (ed.), Ecole d'Été de Probabilités de Saint Flour XIV, 1984, Lecture Notes in Math. 1180. Berlin: Springer-Verlag.

Walsh, J. (1993) Some remarks on $A\left(t, B_{t}\right)$. In J. Azéma, P.A. Meyer and M. Yor (eds), Séminaire de Probabilités XXVII, Lectures Notes in Math. 1557 pp. 173-176. New York: Springer-Verlag.

Yor, M. (1989) Une extension markovienne de l'algèbre des lois béta-gamma. C. R. Acad. Sci. Paris Sér. I Math., 308, 257-260.

Received July 1998 and revised December 1998. 